\title{
Alteraciones psicoemocionales asociadas a la epilepsia en pacientes pediátricos de 8 a 11 años de edad del Hospital Materno Infantil José Domingo de Obaldía
}

\section{Psychoemotional alterations associated to pediatric patients with epilepsy between 8 and 11 years of age at the José Domingo de Obaldía Maternal and Child Hospital}

\author{
Rosalin P. Ríos T. ${ }^{*}$ \\ ${ }^{1}$ Universidad Especializada de las Américas \\ (Dhttps://orcid.org/0000-0002-1962-4441
}

* Autor por correspondencia: Rosalin P. Ríos T. rosalinprios@ hotmail.com

Recibido: 03 de mayo de 2021

Aceptado: 18 de agosto de 2021

\begin{abstract}
Resumen
El presente estudio tuvo el objetivo de analizar las alteraciones psicoemocionales presentes en pacientes pediátricos con epilepsia de ocho a 11 años de edad del Hospital Materno Infantil José Domingo de Obaldía. El diseño de la investigación fue no experimental, transversal. Las alteraciones psicoemocionales estudiadas fueron: ansiedad, depresión, nivel de autoestima, calidad de vida, perturbaciones emocionales inconscientes y conductuales. Se establecieron criterios de inclusión y exclusión para seleccionar la muestra. Se trabajó con un total de 10 pacientes, en el rango de edad indicado; ocho pacientes de sexo masculino y dos del sexo femenino. Para recolectar la información se aplicó a los padres: entrevista clínica semi-estructurada y el Inventario de Calidad de Vida Pediátrica Peds QLA. A los niños se les aplicó: entrevista clínica semiestructurada, Cuestionario de Autoevaluación de Ansiedad Estado-Rasgo en Niños (STAIC), Cuestionario de Depresión para Niños (CDS), Escala de Autoestima del Dr. Cardoze, Inventario de Calidad de Vida Pediátrica Peds QL4 y el Test de Apercepción Infantil con figuras de animales (CAT-A). Los resultados evidenciaron que los pacientes pediátricos con epilepsia presentaban una ligera vulnerabilidad para el desarrollo de alteraciones psicoemocionales, siendo las más relevantes los síntomas ansiosos y depresivos, así como las alteraciones conductuales. Su calidad de vida fue no óptima, el funcionamiento emocional, social y escolar estaban moderadamente afectados. Se reportan problemas para el manejo de emociones, para dormir y rechazo social de compañeros. No se detectó deterioro clínico en la autoestima de los pacientes.

Palabras clave: epilepsia; niños; ansiedad; depresión; autoestima.
\end{abstract}




\begin{abstract}
The present study aimed to analyze the psychoemotional alterations in pediatric patients with epilepsy between 8 and 11 years of age at the José Domingo de Obaldía Maternal and Child Hospital. The research design was non-experimental, cross-sectional. The psychoemotional disorders studied were: anxiety, depression, self-esteem, quality of life, unconscious and behavioral emotional disturbances. Inclusion and exclusion criteria were established to select the sample. We worked with a total of 10 patients in an age range from 8 to 11 years old; eight male patients and two female patients. The collected information, semi-structured clinical interview, and the Pediatric Quality of Life Inventory Peds QL4 were applied to the parents. The children were given: semi-structured clinical interview, State-Trait Anxiety Self-Assessment Questionnaire in Children (STAIC), Depression Questionnaire for Children (CDS), Dr. Cardoze Self-Esteem Scale, Pediatric Quality of Life Inventory Peds QL4, and the Infant Apperception Test with animal figures (CAT-A). The results showed that pediatric patients with epilepsy presented a slight vulnerability to the development of psychoemotional disorders, the most relevant being anxiety and depressive symptoms and behavioral disorders. Their quality of life was not optimal. Their emotional, social, and school functioning were moderately affected-problems with managing emotions, sleeping, and social rejection. No clinical deterioration in the self-esteem of the patients was detected.
\end{abstract}

Keywords: epilepsy; children; anxiety; depression; self-esteem.

\title{
Introducción
}

Las alteraciones psicoemocionales en pacientes pediátricos que padecen enfermedades crónicas son aspectos que han cobrado interés en la psicología en el plano internacional. Se reconoce que las enfermedades crónicas tienen repercusiones importantes en el paciente y la familia (Quesada et al., 2014); sin embargo, gran cantidad de las investigaciones al respecto (Carballeda et al., 2018; Lacomba et al.,2018; Rubio et al., 2010 y Zurita-Cruz et al., 2017) se centran en enfermedades como la diabetes, alergias, asma, obesidad y enfermedades renales en las que se evidencia sintomatología ansiosa y depresiva, dificultades adaptativas y otras alteraciones emocionales relevantes. Por lo tanto, otras enfermedades crónicas pediátricas, como la epilepsia, han sido poco exploradas desde la perspectiva psicoemocional, especialmente en el plano nacional y regional.

Globalmente, la Organización Mundial de la Salud (OMS, 2019) refiere que la epilepsia afecta a más de 50 millones de personas, siendo prevalente en países con ingresos bajos y medianos, de modo que ésta es una problemática evidente en muchos países.

En Panamá, el Ministerio de Salud (MINSA, 2018) en su anuario estadístico, reportó que la población entre cinco y nueve años de edad, se ubicaba, aproximadamente, en 364,928 sujetos; mientras que la población de 10 a 14 años, en 361,445. De ellos, en la provincia de Chiriquí se encuentran 22,092 niños y 21,140 niñas entre cinco y nueve años de edad; 21,810 niños y 20,877 niñas se ubican entre los 10 y los 14 años de edad. Esta información permite comprender la dimensión demográfica de la población pediátrica en el país. El MINSA (2018) en el informe de indicadores de salud básicos, menciona que, aproximadamente, 6,272 personas (hombres y mujeres) padecían de epilepsia para el 2017. Estas cifras no discriminan la cantidad de casos pediátricos. Como se puede observar, en Panamá la base de datos estadísticos puede resultar menos amplia y específica para las enfermedades crónicas, especialmente en la población pediátrica. No obstante, las cifras estadísticas presentadas permiten obtener una visión de cómo es la situación actual de Panamá.

En el ámbito nacional y regional, es notable la frecuencia de pacientes pediátricos que son diagnosticados con enfermedades crónicas como la epilepsia. La aparición, diagnóstico y tratamiento de una enfermedad crónica supone un gran cambio, tanto para el paciente como para la familia debido a las dificultades adaptativas y emocionales que suele implicar. Gómez et al. (2016) menciona que la prevalencia mundial de enfermedades crónicas está en aumento y no se ha investigado a profundidad los problemas mentales que puedan presentar los niños con condiciones crónicas. En consecuencia, resulta necesario estudiar, objetivamente, el ámbito psicológico manifestado en los pacientes pediátricos con enfermedades crónicas, específicamente con epilepsia. De este modo, el problema de investigación se plantea a través de la siguiente pregunta: ¿Cuáles son las alteraciones psicoemocionales más frecuentes en pacientes pediátricos con epilepsia de 8 a 11 años de edad del Hospital Materno Infantil José Domingo de Obaldía?

A partir del planteamiento del problema, se determinan los objetivos. El objetivo general de esta investigación consistió en analizar las alteraciones psicoemocionales presentes en pacientes pediátricos con 
epilepsia de 8 a 11 años de edad del Hospital Materno Infantil José Domingo de Obaldía, distrito de David, provincia de Chiriquí, Panamá, con el fin de conocer la realidad psicoemocional de estos pacientes y resaltar la importancia de la psicología en el tratamiento multidisciplinario de la enfermedad.

\section{Alteraciones psicoemocionales}

Para Fernández y Vílchez (2015) las alteraciones psicoemocionales son estados transitorios que pueden deberse a eventos percibidos como amenazantes por el individuo. Las alteraciones psicoemocionales se conciben como la aparición de emociones percibidas con malestar intenso, que interfieren significativamente en el óptimo desempeño del individuo en distintas áreas, como el ámbito social, escolar, conductual, cognitivo, físico y emocional. Sus manifestaciones son muy variadas, y el evaluador siempre debe considerar la edad del paciente y el contexto sociocultural para estudiarlas.

La entidad Centers for Disease Control and Prevention (2020) resalta que tan solo en Estados Unidos la ansiedad, la depresión y los problemas de autoestima son las alteraciones psicoemocionales más comunes entre los niños y adolescentes. Estos problemas tienden a incrementarse en relación con el aumento de la edad. Bitsko et al. (2018) comentan que se observan incrementos importantes en las cifras de ansiedad y depresión. Así, en niños de seis a 17 años el aumento fue de 5.4\% en el 2003 a 8.4\% entre el 2011 y el 2012; los cambios más significativos ocurrieron en la ansiedad. Ghandour et al. (2019) encontraron que, en Estados Unidos, de los niños entre tres y 17 años de edad, el $7.1 \%$ tiene problemas de ansiedad, un $7.4 \%$ presenta alteraciones conductuales y un $3.2 \%$, depresión.

En Panamá, se carecen de estadísticas longitudinales organizadas sobre la prevalencia de trastornos y problemas en la salud mental pediátrica. Velarde et al. (2014) intentaron establecer la prevalencia de trastornos conductuales y emocionales en la población pediátrica en la ciudad de Panamá en un estudio de carácter transversal, en el que evidenciaron que la ansiedad era uno de los problemas más frecuentes; pues según reportes de los padres, dicha alteración se presentaba en un $8.4 \%$ y conforme con lo encontrado por los maestros, en un $15.3 \%$.

Se reconoce que la ansiedad, depresión, problemas de autoestima y conductuales suelen ser alteraciones psicoemocionales de interés en la población pediátrica (Barrio y Carrasco et al., 2016; Gómez et al., 2016 y Ortuño et al., 2014;), especialmente en aquellas con enfermedades crónicas.

El Manual Diagnóstico y Estadístico de los Trastornos Mentales (2014) define la ansiedad como "una respuesta anticipatoria a una amenaza futura ... a menudo asociada con tensión muscular, vigilancia en relación a un peligro futuro y comportamientos cautelosos o evitativos." (p.189). Se entiende que la ansiedad es una emoción secundaria en la que el sujeto se mueve psicológicamente hacia el futuro y detecta posibles amenazas. La ansiedad es una respuesta normal, que permite la sobrevivencia en un nivel básico, porque posibilita la anticipación a las situaciones peligrosas; pero cuando se experimenta repetidamente y de forma prolongada, adquiere connotaciones desadaptativas y con potencial patológico.

Spielberger (1966) planteó que la ansiedad puede presentarse como estado cuando es transitoria y como rasgo, cuando es indicativo de estabilidad y persistencia como forma de percibir e interpretar la gran mayoría de las situaciones; es decir, un rasgo de la personalidad. Este autor reconoció que la valoración cognitiva tiene un papel importante para comprender el origen, síntomas y control de la ansiedad.

Por otra parte, en relación con la depresión, el Manual Diagnóstico y Estadístico de los Trastornos Mentales (2014) expresa que, aunque existen diversos tipos de trastornos depresivos, hay algunas características comunes, como "la presencia de un ánimo triste, vacío o irritable, acompañado de cambios somáticos y cognitivos que afectan significativamente a la capacidad funcional del individuo." (p. 155). Es necesario diferenciar la tristeza y la depresión. La tristeza es una emoción primaria, transitoria acompañada de respuestas cognitivas, psicológicas y conductuales. Mientras que, la depresión constituye una patología dado que implica la experiencia continua y persistente de tristeza e interfiere en las actividades diarias del sujeto.

Moya y Fernández (2017) engloban las manifestaciones clínicas de depresión de los niños y adolescentes en síntomas: afectivos (estado de ánimo triste, irritabilidad, apatía, anhedonia), físicos (agitación o enlentecimiento psicomotriz, fatiga, astenia, alteraciones del sueño, quejas somáticas y cambios en apetito) y cognitivos (olvidos, dificultad para concentrarse, baja autoestima, ideación suicida pasiva o activa y pensamientos distorsionados). En los niños, la sintomatología depresiva es más variada que en los adultos. El pensamiento y grado de desarrollo emocional del niño están en desarrollo, lo que obstaculiza el reconocimiento y expresión de sus emociones. 
Tanto para la ansiedad como para la depresión, se han desarrollado distintos abordajes teóricos. Pero en la actualidad es el modelo cognitivo el que goza de mayor evidencia científica y aceptación. Este modelo expresa que las alteraciones emocionales no se producen por la situación propiamente, sino por la interpretación cognitiva que atribuye el sujeto. Además, se advierte que alteraciones psicoemocionales, como la ansiedad y la depresión, son respuestas multidimensionales en donde se manifiestan procesos interrelacionados y síntomas cognitivos, fisiológicos, conductuales y afectivos/emocionales (Clark y Beck, 2012; Beck et al. 2010; Lino et al. 2019).

Clark y Beck (2012) describen que el proceso y sostenimiento de la ansiedad sucede a través de la valoración primaria de amenaza y la revaloración secundaria. Las personas con ansiedad tienden a sobrevalorar y sobreestimar la probabilidad de ocurrencia de daños y su intensidad. También minimizan su capacidad de afrontarla y filtran, selectivamente, la información, dando atención a aquellos factores que refuerzan sus esquemas cognitivos de amenaza. Algunos síntomas característicos de la ansiedad son sensación de irrealidad, descenso del razonamiento, tensión muscular, hiperventilación, taquicardia, sudoración, las conductas evitativas, la frustración, impaciencia, entre otros.

En cuanto a la depresión, Beck et al. (2010) explican el modelo cognitivo de la depresión, desarrollado por Beck, en el cual se propone que las personas desarrollan depresión por errores cognitivos sistemáticos en el procesamiento de la información. Para Beck et al. (2010), la tríada cognitiva (visión negativa sobre sí mismo, el futuro y sus experiencias), los esquemas y los errores cognitivos se interrelacionan entre sí, para originar la sintomatología depresiva. En ella se aprecia subestimación de sí mismo, autocrítica, sentimientos de inutilidad, poca valía, procesamiento negativo de experiencias, concepción del mundo como injusto, difícil y hostil; frustración, intensa desesperanza y anticipación negativa del futuro.

En ambas alteraciones psicoemocionales, bajo el modelo cognitivo, se comprende que los patrones cognitivos son aprendidos a lo largo del desarrollo y reforzados por las experiencias negativas, dando lugar al establecimiento de errores sistemáticos de procesamiento de información y creencias rígidas, inflexibles, altamente subjetivas, no realistas y negativas. Es la interpretación negativa, catastrófica y desadaptativa lo que produce el malestar emocional (Beck et al., 2010).

Los indicadores afirmativos para cronicidad de los síntomas, distorsiones cognitivas y deterioro del funcionamiento, pueden denotar anormalidad de la conducta y una sospecha para la presencia de una patología. Por tal motivo, el clínico debe estar atento a los signos y síntomas de los cuadros de los pacientes, especialmente cuando existen diagnósticos de enfermedad crónica. El diagnóstico eficaz y oportuno es esencial en el tratamiento y prevención. Howes et al. (2020) concluyen que la manifestación de ansiedad en edades tempranas incrementa el riesgo de desarrollar psicopatologías, si no recibe intervención.

Asimismo, otros problemas como las alteraciones de la autoestima y conductuales son relevantes en el estudio de la salud mental de los niños, especialmente cuando hay condiciones crónicas. La autoestima es un constructo psicológico que se forma diariamente y que está ligado a la formación del yo, por lo que mantiene implicaciones con el desarrollo psicosocial (Morris y Maisto, 2011). En este proceso la familia, los pares y otros adultos tendrán un papel relevante.

Rosenberg et al. (1995) diferencian dos tipos: autoestima global, relacionada con el bienestar psicológico y la autoestima específica, relacionada con el comportamiento particular. Por su parte, Branden (2007) opina que la autoestima mantiene dos componentes que se relacionan entre sí: la eficacia personal y el respeto por uno mismo. Más recientemente, Mruk (2013) propone que la autoestima tiene dos elementos que se relacionan entre sí: la competencia y el sentido de merecimiento. Reconoce que la autoestima está integrada por cinco componentes: estatus, competencia, retos, merecimiento y tiempo.

Branden (2007) y Roa (2013) enuncian que la autoestima puede encontrarse en niveles básicos en alta y baja. Los problemas de autoestima por sí solos no son una patología en los manuales de diagnósticos, pero representan un punto común en varias patologías. Orth y Robins (2014) indican que los individuos con niveles altos de autoestima, son más proclives a experimentar bienestar en áreas como las relaciones interpersonales, trabajo y salud, a diferencia de los que mantienen niveles bajos de autoestima. Isomaa et al. (2013) señalan que la autoestima está relacionada con síntomas depresivos y ansiosos, por lo que podría considerarse un indicador para varias formas de psicopatología por estudiar.

Cuando existen alteraciones conductuales, también suelen subyacer otras alteraciones psicoemocionales. En niños, las alteraciones conductuales pueden apreciarse en detalles como la apariencia personal, atención, actitud, afecto, lenguaje, desempeño en actividades, reacciones frente a los estímulos y adaptabilidad (Sattler, 2010). Las perturbaciones emocionales, conscientes e inconscientes, influyen en las alteraciones conductuales; pero el estilo 
parental, también. Salari et al. (2014) concretan que los problemas conductuales en los niños están positivamente asociados con las prácticas parentales inefectivas y conflictos entre los padres.

En las alteraciones psicoemocionales se considera la activación conductual de los pacientes debido a que las cogniciones generan emociones y conductas. Así en los niños con síntomas depresivos, se manifestaban conductas de aislamiento social, defensividad o de rebeldía y oposición; en los menores con síntomas ansiosos, puede notarse las dificultades para dormir, comer mucho o perder el apetito, evitar o huir de lo que les preocupa, entre otros (Sánchez y Cohen, 2020).

\section{Epilepsia y alteraciones psicoemocionales}

La OMS (2019) define la epilepsia como una enfermedad crónica de tipo cerebral, caracterizada por crisis convulsivas recurrentes, a causa de descargas eléctricas excesivas de las neuronas, lo que conlleva una amplia variedad de manifestaciones clínicas, conforme con el tipo de crisis y su origen neurológico.

La Liga Internacional contra la Epilepsia (ILAE, 2017) propone que la epilepsia se puede clasificar según su etiología, en: estructural, genética, infecciosa, metabólica, inmunitaria y desconocida. Asimismo, la ILAE (2017) propone que esta patología se clasifique según el tipo de crisis, en: de inicio focal, de inicio generalizado y de inicio desconocido. Conforme con el tipo de epilepsia, se categoriza en: focales, generalizadas, combinadas generalizadas y focales, y desconocidas.

En un primer nivel, el especialista debe reconocer el tipo de crisis y en un segundo nivel, el tipo de epilepsia. En un tercer nivel, se determina el síndrome epiléptico; no obstante, este tercer nivel no siempre es posible establecerlo, debido a las características del caso y el contexto (ILAE, 2017).

En la actualidad, el tratamiento médico predilecto es el farmacológico, como primera opción. Marcia Targas (2014) comenta que los tratamientos farmacológicos antiepilépticos están dirigidos a reducir la intensidad y frecuencia de las crisis. La seguridad del paciente y los efectos adversos son esenciales en el momento en que se elige la opción más óptima. En cuanto a las otras opciones de tratamiento, están los dispositivos implantados (neuromodulación), dietas cetogénicas y la cirugía.

Devinsky y Vazquez (1993) refieren que la depresión, ansiedad e impotencia son problemas frecuentes en pacientes con epilepsia. También se ha demostrado que la epilepsia, tanto en niños como adultos, incluyen síntomas de depresión, ansiedad, miedo, agresividad y dificultades socioadaptativas y que la sintomatología también resulta influenciada por la ubicación del foco epiléptico, el tipo y frecuencia de las crisis (Ramos-Loyo y Sanz-Martin, 2005). Los niños y adolescentes con epilepsia del lóbulo temporal exhibían tasas más altas de depresión con respecto a los que tenían epilepsia del lóbulo frontal (Schraegle y Titus, 2017).

Otros estudios (Baki et al., 2004; Kotwas et al., 2017; Seyfhashemi y Bahadoran, 2013) evidencian que los pacientes con epilepsia son más propensos a desarrollar perturbaciones emocionales, siendo la sintomatología depresiva la más frecuente, seguida de la ansiedad. Larson et al. (2012) apuntan que la epilepsia pediátrica afecta los patrones de sueño y conductas al dormir, del paciente y sus padres.

De esta manera, la salud mental y calidad de vida del paciente y familia resultan impactadas en distintas áreas. Recientemente, la Liga Internacional contra la Epilepsia y varios investigadores se han interesado por este tema. Michaelis et al. (2018) señalan que las intervenciones psicológicas deben ser parte del manejo integral del paciente con epilepsia, ya que incrementa la adherencia al tratamiento y disminuyen la ocurrencia de síntomas ansiosos y depresivos.

\section{Materiales y Método}

\section{Objetivos}

El tipo de investigación obedeció a un enfoque mixto y, según su alcance, fue descriptivo. El diseño de la investigación fue no experimental transversal. El objetivo general de esta investigación consistió en analizar las alteraciones psicoemocionales presentes en pacientes pediátricos con epilepsia de 8 a 11 años de edad del Hospital Materno Infantil José Domingo de Obaldía, distrito de David, provincia de Chiriquí, Panamá. Adicionalmente, se establecieron los objetivos específicos de identificar las características psicoemocionales que presentan los pacientes pediátricos diagnosticados con epilepsia, describir los principales tipos de alteraciones 
psicoemocionales que manifiestan y, finalmente, señalar cómo se manifiestan las alteraciones psicoemocionales en los pacientes pediátricos con epilepsia.

\section{Población y muestra}

El universo de estudio de este trabajo investigativo correspondió a los pacientes pediátricos del Hospital Materno Infantil José Domingo de Obaldía, diagnosticados con epilepsia, que acudían al servicio de psicología de esta institución, en David, provincia de Chiriquí, Panamá.

El método de muestreo seleccionado fue no probabilístico, por conveniencia. Se trabajó con aquellos casos que fueron referidos por neuropediatría, con diagnóstico de epilepsia y que cumplían con los criterios durante el período de recolección de la información en la institución (agosto-octubre 2020). Se establecieron criterios de inclusión y exclusión.

Durante el desarrollo de este estudio, se trabajó con un total de 10 pacientes, en un rango de edad de ocho a 11 años, ocho de sexo masculino y dos de sexo femenino. De los casos estudiados, el $80 \%$ correspondió a epilepsia generalizada y el $20 \%$ restante, a epilepsia focal. Este diagnóstico fue establecido por neuropediatría, facilitado por los padres y confirmado con el historial del paciente. Igualmente, es necesario resaltar que, aproximadamente, el 50\% de los pacientes tenía antecedentes familiares de epilepsia, lo que refuerza el hecho de la predisposición genética para el desarrollo de esta patología.

\section{Instrumentos}

Los instrumentos seleccionados para la investigación fueron aplicados a los padres y a los niños participantes. Se aplicaron los siguientes instrumentos a los padres: entrevista clínica semi estructurada y el Inventario de Calidad de Vida Pediátrica Peds QL4-informe de los padres. A los pacientes pediátricos se les aplicó: entrevista clínica semi estructurada, Cuestionario de Autoevaluación de Ansiedad Estado-Rasgo en Niños (STAIC), Cuestionario de Depresión para Niños (CDS), Escala de Autoestima del Dr. Cardoze, Inventario de Calidad de Vida Pediátrica Peds QL4 y el Test de Apercepción Infantil con figuras de animales (CAT-A).

La entrevista clínica semi estructurada, según Satler (2010), está conformada por una lista de preguntas cuyo enfoque y profundidad puede variar dependiendo de la información suministrada. Permite conocer los antecedentes personales y familiares, hábitos, conductas, estilo de disciplina, datos del neurodesarrollo y diversos hitos del desarrollo en sus diversas áreas.

El Inventario de Calidad de Vida Pediátrica Peds QL4, inicialmente la prueba se utilizaba para pacientes pediátricos con cáncer en Estados Unidos (Varni, Katz, Seid, Quiggins y Friedman-Bender, 1998). La escala cuenta con una versión para los padres y otra para el niño, evaluando los mismos aspectos. El instrumento está compuesto por 23 ítems, distribuidos en cuatro áreas de funcionamiento: físico, emocional, social y escolar, permitiendo la medición de la percepción de los padres sobre las diversas áreas de funcionamiento de su hijo y la del propio niño, en donde, 0 (nunca es un problema), 1 (casi nunca es un problema), 2 (algunas veces es un problema), 3 (con frecuencia es un problema) y 4 (casi siempre es un problema). Para el cálculo de la medida de funcionamiento por área, se realiza la transformación lineal, conforme al autor del instrumento, de los ítems de respuesta a una escala de $0-100(0=100,1=75,2=50,3=25,4=0)$. El cálculo de las puntuaciones de las dimensiones se realiza dividiendo la suma de las puntuaciones de los ítems correspondientes a la sección, entre el número de ítems respondidos por cada área de funcionamiento. No hay puntuaciones de corte, las puntuaciones oscilan de 0-100. A puntuaciones más bajas, peor funcionamiento y calidad de vida. El uso de este instrumento requirió la autorización facilitada por Mapi Research Trust, localizado en Lyon, Francia y del investigador y autor del instrumento utilizado James W. Varni, PhD.

Sobre el Cuestionario de Autoevaluación de Ansiedad Estado-Rasgo en Niños (STAIC) Spielberger et al. (2009) especifican que la prueba mide el factor de la ansiedad en sus dos modalidades: estado; es decir, de carácter transitorio y como rasgo, carácter permanente en la personalidad del individuo. Está conformada por 40 ítems en total, 20, para ansiedad estado y 20, para ansiedad rasgo. Las puntuaciones para cada una oscilan entre 20 a 60 puntos. De acuerdo a los baremos establecidos en la prueba, las puntuaciones percentiles significan: bajo (1-15), promedio bajo (16-49), promedio (50), promedio alto (51-84) y alto (85-99).

El Cuestionario de Depresión para Niños (CDS) diseñado por Lang y Tisher (2003) permite la evaluación global y específica de la depresión en niños. Está conformado por 66 elementos, la evaluación específica de la depresión se obtuvo por las subescalas que constituyen las dimensiones globales de total depresivo y total 
positivo. El total depresivo está compuesto de las subescalas de respuesta afectiva, problemas sociales, autoestima, preocupación por la muerte/salud, sentimiento de culpabilidad y depresivos varios. El total positivo lo forman las subsescalas de ánimo alegría y positivo varios. Las puntuaciones en decatipos, de acuerdo a los baremos sugieren: bajo (1-3), promedio bajo (4), promedio (5-6), promedio alto (7), alto (8-10).

La Escala de Autoestima del Dr. Cardoze, diseñada por Cardoze (2005), es escala auto-evaluativa que permite detectar problemas de autoestima a través de 14 ítems. Sus puntaciones indican: muy alta (87-100), alta (73-86), normal (59-72), baja (45-58) y muy baja (0-44).

El Test de Apercepción Infantil con figuras de animales (CAT-A) es una prueba psicológica cualitativa de carácter proyectivo diseñada por Bellak y Sorel Bellak (2007) que permite explorar la relación de un niño con sus figuras más importantes, lo que posibilita conocer aspectos diversos como actitud frente a los padres, rivalidad fraternal, problemas de alimentación, miedos, autoimagen, entre otros.

\section{Procedimiento de recogida y análisis de datos}

La investigación cumplió con los principios éticos y morales que rigen las investigaciones con seres humanos: Declaración de Helsinki, Informe Belmont, Buenas Prácticas Clínicas y las normas y criterios éticos establecidos en Panamá. La investigación recibió la aprobación del Comité de Bioética de la Universidad de Santander, ciudad de Panamá.

El proceso de recogida de la información guardó el cumplimiento de la confidencialidad y las normas de bioética; se explicó y presentó el consentimiento informado a los padres, mientras que, a los menores, el asentimiento informado. Posteriormente, se procedió a la recolección de la información, mediante la aplicación de los instrumentos a padres y niños en, aproximadamente, cuatro a cinco sesiones individuales, con un máximo 60 minutos.

En psicología, el proceso de evaluación y análisis de un paciente obedece a la aplicación de los cuatro pilares: entrevista clínica, pruebas estandarizadas, pruebas subjetivas y observaciones conductuales. En la presente investigación, se desarrolló el análisis de resultados de forma integral, involucrando, según el instrumento, información psicométrica y cualitativa. Los instrumentos psicométricos gozaban de validez y confiabilidad, posibilitando la interpretación de las puntuaciones con referencia a la norma. Los resultados de estas pruebas fueron procesados de acuerdo con las especificaciones de cada test, en donde de forma general, se obtuvo una puntuación directa, que luego fue transformada e interpretada según los baremos correspondientes y el manual del test. Las pruebas cualitativas brindaban detalles descriptivos complementarios sobre lo evaluado en el paciente.

Además, se puntualiza que el programa de análisis de los datos seleccionado fue el programa Microsoft Excel. Este programa cuenta con múltiples herramientas, que facilitaron los cálculos matemáticos y estadísticos de frecuencias absolutas, frecuencias porcentuales y promedios, así como con la capacidad para elaborar tablas y gráficas necesarias para organizar los resultados.

\section{Resultados}

Los resultados de los datos generales que permiten comprender el contexto de la información respecto al nivel de escolaridad. Un $40 \%$ de los participantes cursaba el tercer grado; otro 40\%, el cuarto grado, mientras que un $10 \%$, el primer y sexto grado, respectivamente. La mayoría de estos pacientes presentaban dificultades para la lectoescritura, evidenciadas durante las sesiones y referenciada por los padres en la entrevista clínica.

\section{Resultados psicométricos}

La ansiedad fue evaluada por medio del Cuestionario de Autoevaluación de Ansiedad Estado-Rasgo en Niños (STAIC). Este instrumento permitió evaluar la ansiedad como estado y rasgo. Como se puede observar en la Figura 1, en la dimensión de ansiedad estado, el $80 \%$ de los pacientes puntuaron promedio bajo, el resto se ubicó en promedio y bajo. Esto sugiere que los pacientes, en el momento de la evaluación, no se encontraban en cuadro activo de ansiedad temporal y transitoria.

Por otra parte, en la Figura 1, para la dimensión de ansiedad rasgo, las puntuaciones no estaban tan concentradas: un $40 \%$ mantenía niveles altos; un $20 \%$ se encontraba en promedio alto; un $10 \%$ estaba en nivel promedio; un $20 \%$, promedio bajo y un $10 \%$, nivel bajo. Estos pacientes presentaban una ligera tendencia a 
manifestar la ansiedad como un rasgo estable de su personalidad. Según el análisis psicológico de las dimensiones evaluadas por la prueba, los ítems comunes en casi todos los pacientes estuvieron relacionados con indecisión y mucha preocupación por lo que pueda ocurrir y acerca de los errores que puedan cometer.

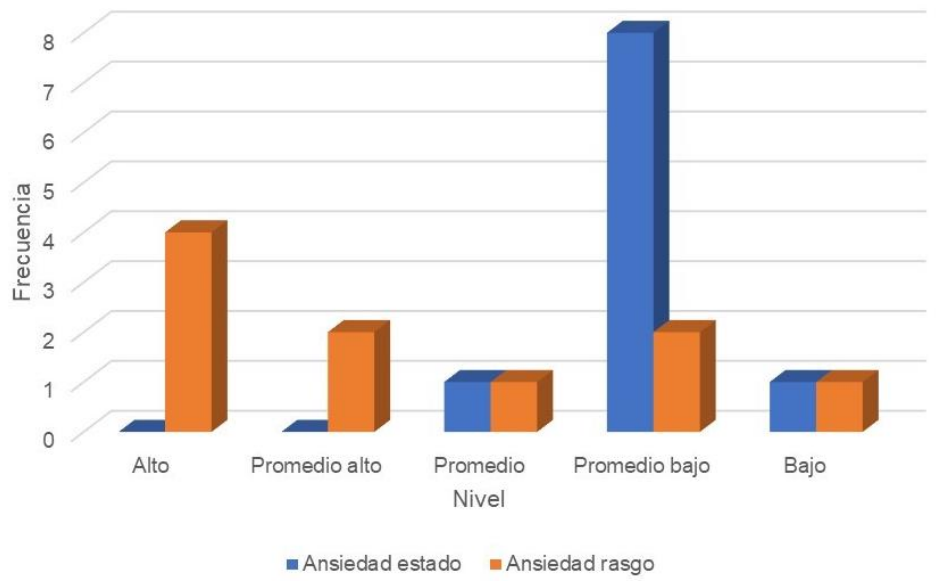

Figura 1. Niveles de ansiedad estado-rasgo

La depresión fue evaluada a través del Cuestionario de Depresión para Niños (CDS). Este test psicométrico brindaba puntuaciones en dos dimensiones globales: el total depresivo y el total positivo. Cada una de ellas estaba conformada por otras subescalas. Primero, se presenta el resultado de las dimensiones globales (Figura 2) y luego, las subescalas (Tabla 1), según los niveles de la prueba.

El total depresivo, de acuerdo a Lang y Tisher (2003), es la dimensión compuesta por las respuestas afectivas negativas, problemas sociales del niño, el aislamiento, sentimientos de soledad, problemas de autoestima, preocupaciones por la muerte o la salud, sentimientos de culpabilidad y autopunitivos. En esta dimensión, se encontró que el $50 \%$ de los participantes mantenía puntuaciones promedio alto, un 20\% tiene puntuaciones altas; mientras que el otro $30 \%$ restante se encontraba distribuido en un $10 \%$ en las puntuaciones bajo, promedio bajo y promedio como se puede apreciar en la Figura 2.

El total positivo se relaciona con la incapacidad o dificultad del niño para experimentar y vivenciar alegría, diversión y felicidad (Lang y Tisher, 2003). En esta dimensión, tal como se muestra en la Figura 2, el 80\% de los niños presentó un nivel promedio alto y un $20 \%$, nivel alto. Este es otro indicador importante para la sintomatología depresiva, ya que demostró que a la mayoría de estos niños se les dificultaba experimentar emociones, como la alegría y felicidad, o las experimentaban con menos frecuencia en relación con el promedio de los niños de su edad.

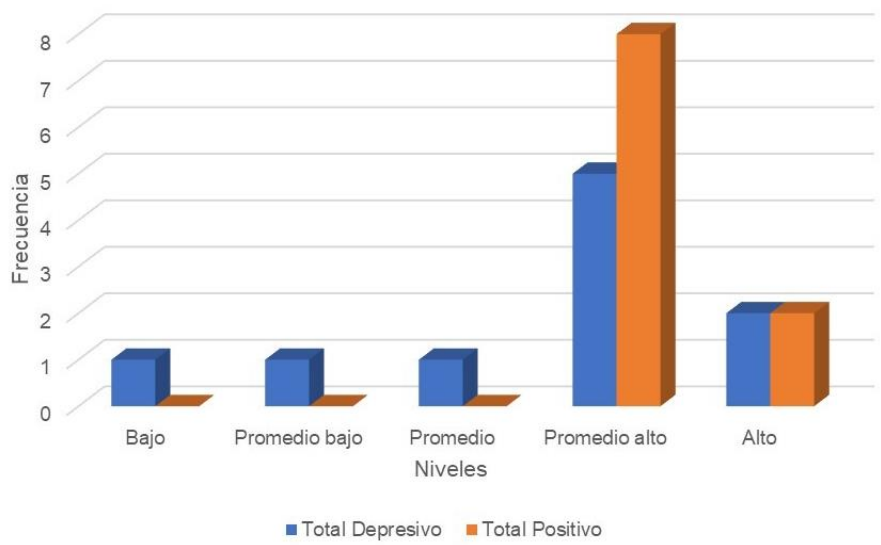

Figura 2. Dimensiones globales para el Cuestionario de Depresión para Niños 
Con respecto al Cuestionario de Depresión para Niños, también se obtenían puntuaciones por subescala para la evaluación específica de la depresión. En detalle, se inicia a exponer los resultados catalogados como nivel alto: preocupación por la muerte/salud, detectado en el $40 \%$ de la muestra en nivel alto.

Dentro de los que se manifestaron en el $50 \%$ o más de la población estudiada, estaban los que se ubican en nivel promedio alto: problemas sociales y positivos varios, seguido de respuesta afectiva, autoestima, preocupación por la muerte/salud, sentimiento de culpabilidad y depresivos varios, como se evidencia en la Tabla 1.

Las subscalas más recurrentes, mayormente las de nivel promedio alto, significaban que los participantes de la muestra desarrollan síntomas depresivos con una incidencia ligeramente superior al promedio. Los síntomas depresivos de mayor relevancia clínica, según las respuestas de los pacientes en la prueba, estaban vinculados con tendencia al aislamiento, sentimientos de soledad, sensación de poco merecimiento de afecto, pérdida de energía y cansancio, necesidad de validación y aprobación de sus figuras parentales, percepción de la vida como sufrida, preocupaciones por su salud y la de su figura maternal.

También manifestaron inquietud con respecto a la percepción de sus padres sobre ellos y sus acciones (terribles o buenas acciones), sentirse mal por no amar y escuchar a sus padres como merecen, dificultades para mantener el sueño en la noche y sensación de no ser lo suficientemente bueno.

En este desglose por subescalas, se nota que las puntuaciones promedio alto fueron más predominantes para todas las subescalas, como se muestra en la Tabla 1.

Tabla 1. Subescalas del Cuestionario de Depresión para Niños

\begin{tabular}{lccccc}
\hline \multicolumn{1}{c}{ Subescala } & \multicolumn{5}{c}{ Niveles } \\
\cline { 2 - 6 } & Bajo & Promedio bajo & Promedio & Promedio alto & Alto \\
\hline Respuesta afectiva (RA) & 1 & 2 & 0 & 5 & 2 \\
Problemas sociales (PS) & 1 & 1 & 0 & 6 & 2 \\
Autoestima (AE) & 1 & 3 & 0 & 5 & 1 \\
Preocupación por la muerte/salud (PM) & 1 & 0 & 0 & 5 & 4 \\
Sentimiento de culpabilidad (SC) & 0 & 4 & 1 & 5 & 0 \\
Depresivos varios (DV) & 2 & 1 & 0 & 5 & 2 \\
Ánimo-alegría (AA) & 0 & 1 & 3 & 4 & 2 \\
Positivos varios (PV) & 0 & 0 & 1 & 6 & 3 \\
\hline
\end{tabular}

Como se puede ver en la Figura 3, el $40 \%$ de los niños presentó una autoestima alta; un 20\%, autoestima normal y otro $20 \%$, autoestima baja. En las puntuaciones extremas se encontraba un $10 \%$ con autoestima muy alta y otro $10 \%$ con autoestima muy baja.

Se comprende que la autoestima del promedio de los niños no estaba significativamente afectada. Sin embargo, es necesario reconocer que tanto en los que obtuvieron puntuaciones altas como bajas, existen ciertos aspectos comunes en los que se evidenció malestar o dificultad. Algunos de estos aspectos fueron los relacionados con el sentido de reconocimiento y percepción de las figuras parentales, como ser considerado buen hijo y hacer felices a sus padres; otros vinculados con el autoconcepto y autoeficacia, como la valoración de sus capacidades intelectuales para ser buen alumno, tener buenas ideas y juicios razonables adecuados. 


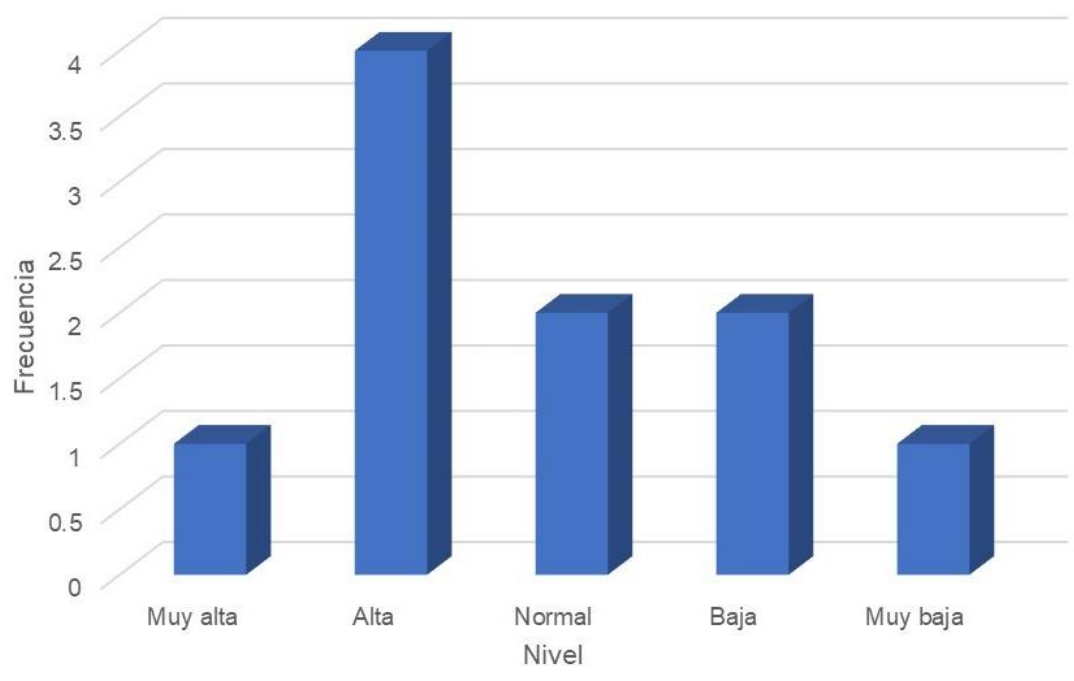

Figura 3. Niveles de autoestima

\section{Resultados cualitativos}

A continuación, se presentan los resultados cualitativos de la investigación, obtenidos mediante la entrevista clínica semi-estructurada, el Inventario de Calidad de Vida Pediátrica Peds QL4, las observaciones conductuales y el Test de Apercepción Infantil con figuras de animales (CAT-A).

La evaluación de la calidad de vida indicó el nivel de funcionamiento por área. Este nivel fue establecido conforme con las indicaciones del autor del instrumento, en una línea de 0 a 100. En la Tabla 2, se observan las puntuaciones promedio del Inventario de Calidad de Vida Pediátrica Peds QL4, según las respuestas brindadas por los niños. En general, las puntuaciones no revelaban afectaciones significativas, pero no reflejaban un nivel óptimo de calidad de vida.

Tabla 2. Calidad de vida según niños

\begin{tabular}{lcc}
\hline Área de funcionamiento & Promedio (\%) & Principales problemas \\
\hline Físico & 73.4 & -Ayudar en las tareas de la casa. \\
\hline Emocional & 62.5 & -Preocupaciones por lo que sucederá en el futuro y problemas para dormir. \\
\hline Social & 61.5 & -Otros niños no quieren ser sus amigos. \\
\hline Escolar & 49.0 & -Faltar a menudo a la escuela por ir al doctor y al hospital. \\
\hline
\end{tabular}

El funcionamiento biopsicosocial fue un reflejo de la calidad de vida percibida por el paciente pediátrico. Tal como se refleja en los resultados, su calidad de vida no es excelente, pero tampoco pésima. Se encontraron alteraciones leves, que deben ser atendidas, especialmente el funcionamiento emocional, social y escolar.

Con respecto a la calidad de vida según los padres, en general, se apreció una diferencia significativa con respecto a las puntuaciones de lo reportado por los niños. Las valoraciones de los padres sobre el funcionamiento biopsicosocial mostraron un detrimento importante, aunque revelaron la misma tendencia que en los reportes de los niños, siendo el funcionamiento emocional, social y escolar los que se hallaban más afectados, tal como se observa en la Tabla 3. El absentismo escolar por citas y monitoreo médico fue coincidente con lo referido por los niños. 
Tabla 3. Calidad de vida según padres

\begin{tabular}{lcc}
\hline Área de funcionamiento & Promedio (\%) & \multicolumn{1}{c}{ Principales problemas } \\
\hline Físico & 47.5 & -Ayudar en casa y hacer deportes o ejercicios. \\
\hline Emocional & 38.5 & -A menudo sus hijos se sienten tristes o apenados, con miedo o susto. \\
\hline Social & 35.0 & -Otros niños se burlan del menor. \\
\hline & 32.5 & -Olvidar las cosas, poner atención en clase, mantenerse al día en las tareas \\
Escolar & & escolares y faltar a la escuela por ir al doctor/hospital. \\
\hline
\end{tabular}

Por otra parte, las observaciones conductuales fueron abastecidas por todo el proceso de evaluación; pero, principalmente, por la entrevista clínica semi-estructurada a padres y niños, así como por el comportamiento. En la Tabla 4, se describen las alteraciones conductuales observadas.

Tabla 4. Alteraciones conductuales observadas

\begin{tabular}{|c|c|}
\hline Área & Niños \\
\hline $\begin{array}{l}\text { Actitud ante el } \\
\text { evaluador }\end{array}$ & $\begin{array}{l}\text { La mayoría de los niños fueron colaboradores. Cooperaban y seguían las indicaciones en el } \\
\text { desarrollo de las actividades y respetaban las figuras de autoridad; sin embargo, antes de iniciar la } \\
\text { aplicación de pruebas, fue necesario crear un buen rapport para favorecer la comunicación de los } \\
\text { aspectos emocionales. }\end{array}$ \\
\hline Afectividad & $\begin{array}{l}\text { Todos los evaluados respondían adecuadamente a los estímulos motivacionales y afectivos } \\
\text { brindados. No obstante, en el proceso de evaluación, la mayoría evidenció dificultad para expresar } \\
\text { y manejar sus emociones, especialmente el enojo, tristeza y frustración. } \\
\text { Se observó preocupación por el futuro sobre su salud, familia y escuela, así como el sentimiento de } \\
\text { pena por no poder hacer lo que otros niños hacen fácilmente. } \\
\text { Estas observaciones también fueron señaladas por los padres; es decir, que son observables en } \\
\text { distintos entornos. }\end{array}$ \\
\hline $\begin{array}{l}\text { Conducta } \\
\text { trabajo }\end{array}$ & $\begin{array}{l}\text { La mayoría de los participantes reflejaron inquietud psicomotora, dificultad para permanecer en el } \\
\text { puesto, cambios de posturas frecuentes y conducta de trabajo inadecuada, caracterizada por } \\
\text { impulsividad y organización regular. } \\
\text { En gran parte de los evaluados, se detectó baja tolerancia a la frustración y dificultades para mantener } \\
\text { la atención. Se distraen fácilmente, por lo que necesitan de la motivación y guía del adulto durante } \\
\text { el desarrollo de las actividades, para lograr un mejor rendimiento. } \\
\text { Las dificultades en atención sostenida fueron bastante notorias y expresadas por los padres. }\end{array}$ \\
\hline Cognitiva & $\begin{array}{l}\text { Gran parte de los niños evaluados presentaron dificultades importantes en las actividades de lecto- } \\
\text { escritura, de manera que requirieron de ayuda adicional en los estímulos que involucraban lectura. } \\
\text { Los déficits en lecto-escritura no estaban acordes con lo esperado para su edad cronológica, y ante } \\
\text { esas dificultades, los niños reflejaron incomodidad, inseguridad y pena. } \\
\text { Los padres de los menores también refirieron dichas dificultades en el trabajo, en casa y escuela. }\end{array}$ \\
\hline $\begin{array}{l}\text { Conductas de } \\
\text { independencia }\end{array}$ & $\begin{array}{l}\text { La mayoría de los niños mostraron conductas dependientes de un adulto o tercero, para lograr } \\
\text { desarrollar exitosamente las actividades. } \\
\text { Sobre el autocuidado, reconocieron qué actividades pueden emprender sin que afecte su salud. Ante } \\
\text { la medicación, casi todos los evaluados no manifestaron rechazo frente a la ingesta de los fármacos. } \\
\text { Estos son administrados regularmente por el cuidador principal. Los tutores coinciden en estar } \\
\text { pendientes de la toma de los medicamentos y que, en ocasiones, perciben frustración en sus hijos, } \\
\text { por lo impredecible de las crisis. }\end{array}$ \\
\hline
\end{tabular}

Las perturbaciones emocionales inconscientes fueron evaluadas por el Test de Apercepción Infantil con figuras de animales (CAT-A). El análisis proporcionado en esta sección, Tabla 5, está centrado en las características comunes de los pacientes y no en sus diferencias particulares. 
Tabla 5. Análisis del Test de Apercepción Infantil con figuras de animales (CAT-A)

\begin{tabular}{|c|c|}
\hline $\begin{array}{c}\text { Aspecto } \\
\text { explorado }\end{array}$ & Interpretaciones \\
\hline Temas recurrentes & $\begin{array}{l}\text {-Necesidad de recibir cuidados y gratificaciones por parte de la madre. } \\
\text {-Celos fraternales, percepción de la madre como autoritaria y controladora, dificultad con las } \\
\text { conductas de independencia, competencia con la figura paternal, sentimientos de culpa y marcada } \\
\text { ansiedad. }\end{array}$ \\
\hline $\begin{array}{l}\text { Necesidades } \\
\text { predominantes }\end{array}$ & $\begin{array}{l}\text {-Ser cuidados, protegidos, gratificados y amados por su madre, siendo ellos los únicos en recibir los } \\
\text { afectos maternales. } \\
\text {-Coexistencia de deseos de independencia. }\end{array}$ \\
\hline $\begin{array}{l}\text { Concepción del } \\
\text { mundo }\end{array}$ & -Lugar hostil, agresivo, peligroso e inseguro, que no podrían enfrentar por sí solo \\
\hline $\begin{array}{l}\text { Relación con los } \\
\text { demás }\end{array}$ & $\begin{array}{l}\text {-Necesidad de tener el apoyo de otros para crecer, cuando se sienten inseguros, y cierta tendencia a } \\
\text { la dependencia. }\end{array}$ \\
\hline $\begin{array}{l}\text { Conflictos más } \\
\text { significativos }\end{array}$ & $\begin{array}{l}\text {-Lucha entre el superyó versus el ello; la agresividad reprimida, dependencia materna, celos } \\
\text { fraternales, angustia por el abandono de los padres y pulsiones sexuales. } \\
\text {-Angustias sexuales y deseos epistemofílicos (deseo de saber más, particularmente los secretos de los } \\
\text { adultos). }\end{array}$ \\
\hline $\begin{array}{l}\text { Naturaleza de las } \\
\text { ansiedades }\end{array}$ & $\begin{array}{l}\text {-Desaprobación materna, temores hacia el daño físico como producto de sus pulsiones y la } \\
\text { competencia; falta o pérdida de cariño de sus figuras de apego; temor a no ser amado lo suficiente, a } \\
\text { ser abandonado y a no ser lo suficientemente bueno para los padres, así como a expresar totalmente } \\
\text { su agresividad. }\end{array}$ \\
\hline $\begin{array}{l}\text { Principales } \\
\text { defensas }\end{array}$ & $\begin{array}{l}\text {-Represión, personificación, vuelta contra sí mismo, racionalización, proyección de la agresividad, } \\
\text { sublimación, actitudes de dependencia, negación y conductas evasivas. En una menor medida, el uso } \\
\text { de la disociación. }\end{array}$ \\
\hline Superyó & -Integrado. \\
\hline
\end{tabular}

\section{Discusión y conclusiones}

Conforme con los resultados obtenidos, los pacientes pediátricos con epilepsia estudiados presentan una ligera susceptibilidad en relación con el promedio de los niños de su edad cronológica, para el desarrollo de alteraciones psicoemocionales, de las cuales las más relevantes son los síntomas ansiosos, depresivos y alteraciones conductuales. Estas alteraciones psicoemocionales leves no corresponden a un estado transitorio, sino a un patrón de respuesta cognitivo-emocional-conductual ante las situaciones, lo que aumenta el grado de vulnerabilidad para el desarrollo de alteraciones emocionales significativas en estadios posteriores del desarrollo. Cabe resaltar que los resultados obtenidos son congruentes con las investigaciones realizadas en otros países en este tipo de pacientes, quienes manifestaban más síntomas depresivos, ansiosos y dificultades socioadaptativas (Baki et al., 2004; Devinsky y Vazquez, 1993, 2003; Kotwas et al., 2017; Ramos-Loyo y Sanz-Martin, 2005; Seyfhashemi y Bahadoran, 2013).

Kotwas et al. (2017) señalan que los pacientes con epilepsia suelen manifestar depresión y ansiedad, debido a la impredecibilidad de las convulsiones y a las múltiples limitaciones que representa la enfermedad, que suele acarrear estigmatización social, situaciones que también fueron evidenciadas en esta investigación, dado que los pacientes que tuvieron una crisis epiléptica en el aula de clases reportaron que sus compañeros mostraban actitud de miedo y rechazo hacia ellos.

Los resultados de la presente investigación revelan que los síntomas ansiosos predominantes fueron la preocupación por los eventos que sucederán en el futuro, la indecisión y el cometer errores. Las preocupaciones están en torno a la salud, tanto la propia como la de su familia, hecho que se realimenta por lo impredecible de las crisis epilépticas. Esto indica que los pacientes con epilepsia enfrentan mayor incertidumbre en el diario vivir, por lo que hay mayor riesgo de que la tendencia ansiosa se asiente como una forma de procesamiento y de respuesta relativamente estable en la personalidad del sujeto. Estos hallazgos son compatibles con lo que comentan Wei et al. (2021) acerca de que la preocupación intensa es un síntoma de ansiedad usualmente presente en las personas con epilepsia.

Con respecto a los síntomas depresivos evaluados, los pacientes evidencian una mayor propensión a manifestar este tipo de síntomas en comparación con el promedio de los niños de su grupo etario por sus puntuaciones mayoritarias en el nivel promedio alto y alto para las dimensiones de total depresivo y positivo 
obtenidas en el instrumento aplicado; sin embargo, no son de carácter clínico para representar, en estos momentos, un diagnóstico de depresión. Los síntomas depresivos más relevantes fueron la dificultad de los niños para experimentar alegría, preocupaciones por su salud y la de su madre, sentimientos de soledad, tendencia al aislamiento, sentimientos de inferioridad, percepción negativa de la vida, entre otros. Según Beck et. al (2010) los sujetos con tendencia depresiva denotan una visión negativa sobre sí mismo, el futuro y sus experiencias, elementos que se encontraron en los participantes del estudio. Wei et al. (2021) identifica el estado de ánimo triste y la sensación de inutilidad reportada en los pacientes con epilepsia como síntomas de especial atención, especialmente por su asociación con la ideación suicida. La sintomatología depresiva evidenciada afecta los comportamientos de los niños en el nivel personal y social, de manera que, pese a no constituir una patología depresiva en el momento actual, el presentar síntomas depresivos como los descritos, constituye una tendencia riesgosa en etapas posteriores del desarrollo. Baki et al. (2004) evidenció que los niños con epilepsia presentan más frecuentemente sintomatología depresiva que otros niños. Schraegle y Titus (2017) enfatizan en que la epilepsia pediátrica se observa una prevalencia significativa de rasgos psicopatológicos.

Según la sintomatología ansiosa y depresiva encontrada en los niños con epilepsia estudiados se observa que presentan más vulnerabilidades para el desarrollo de psicopatologías. Al respecto, Jones (2014) enfatiza que los pacientes pediátricos presentan un riesgo significativo para comorbilidades psiquiátricas como el trastorno de déficit de atención/hiperactividad, depresión y ansiedad. Christensen et al. (2007) identifica a los pacientes con diagnóstico reciente de epilepsia como un grupo vulnerable y con riesgo más elevado de suicidio.

En la autoestima no se detectó deterioro consistente entre los pacientes; la mayoría mantenía un nivel de autoestima dentro del promedio. Mientras tanto, la calidad de vida reportada por los pacientes pediátricos y padres reveló un funcionamiento biopsicosocial afectado; de forma leve, según las respuestas de los niños, y moderada, de acuerdo con las contestaciones de sus padres. Las áreas de funcionamiento más alteradas han sido el funcionamiento emocional, social y escolar. Kotwas et. al (2017) expresan que el deterioro en la calidad de vida del paciente suele ser mayor en el funcionamiento social. En el reporte de los padres sobre sus hijos, se evidencian alteraciones en el funcionamiento escolar, social y emocional, vinculadas al manejo de las emociones. Otra dificultad consistente fue los problemas para dormir. Esto coincide con los hallazgos realizados por Larson et al. (2012), quienes confirmaron que los niños que padecen epilepsia presentaban una mayor disfunción del sueño infantil; mientras más grave fuera la epilepsia, mayor alteración del sueño. Zambrelli et al. (2020) en su investigación coincide en que los desórdenes en el sueño, comportamiento y estado de ánimo son más comunes en la epilepsia en niños que aquellos que no padecen de la enfermedad. Sus resultados arrojan puntuaciones anormales para el sueño en un $23.8 \%$ de los participantes en comparación con el grupo control. Bragantini et al. (2019) advierte que los niveles de ansiedad eran más altos en los individuos que reportaban síntomas de insomnio, en comparación con los controles, por lo que podría haber una interrelación entre síntomas de insomnio y ansiedad.

En general, la calidad de vida percibida por los niños y los padres muestra un detrimento global en la puntuación. Diversas investigaciones revelan que la epilepsia pediátrica tiene un impacto en la reducción de la percepción de la calidad de vida (López et al., 2010; Nadkarni et al., 2011; Conde et. al., 2020).

Dentro de las alteraciones psicoemocionales, se denotan alteraciones conductuales en la mayoría de los pacientes, entre las que se encuentran la dificultad para mantener la atención, conductas de trabajo inadecuadas, dificultades en el manejo y expresión de sus emociones, dependencia y dificultades en la lectoescritura. Karanja et al. (2021) reporta que, en su investigación, detectó en un $46 \%$ de los participantes problemas emocionales y de comportamiento, siendo los problemas de atención, comportamiento agresivo, sociales y de retiro/depresión los más notables. Dal Canto et al. (2017) según sus hallazgos también coincide en que los niños con epilepsia tienen mayor riesgo de desórdenes emocionales y del comportamiento.

Los resultados del análisis del CAT-A se complementaron cualitativamente con los demás instrumentos aplicados en esta investigación. Por ejemplo, en la dificultad para reconocer y expresar sus emociones, se apreció la represión; en las alteraciones conductuales manifestadas se denotaron conductas evasivas y dependientes de la figura que provee seguridad, primordialmente sus figuras de apego, quienes le proveen ayuda y soporte. En las sesiones de evaluación, se presentó como la necesidad constante de guía por parte del evaluador para culminar satisfactoriamente las tareas, ya que necesitaron mayor guía que otros niños de su edad cronológica. Otras alteraciones psicoemocionales exhibidas que mostraron las perturbaciones emocionales inconscientes, fueron los síntomas depresivos leves en el promedio de los niños. Cualitativamente, en el CAT-A se apreció la vuelta contra uno mismo (agresión internalizada hacía sí mismo), represión, negación y concepción negativa del 
mundo.

En este mismo instrumento se encontraron conductas evasivas, que aumentan la ansiedad, la dependencia de las figuras de apego para manejar situaciones conflictivas, la proyección de la agresividad y los temores diversos asociados a no ser lo suficientemente bueno para sus padres, al abandono, la pérdida del cariño, la desaprobación materna e inconscientemente, a la expresión de su agresividad reprimida y a las demandas de su superyó. En la ansiedad, el individuo tiende a minimizar sus recursos de afrontamiento (Clark y Beck, 2012). De igual manera, las conductas evasivas, dependientes y la necesidad de ser cuidado o protegido, que se refuerza por la epilepsia, afectan el funcionamiento social de los niños, ya que las estrategias de evitación y la tendencia a ser sobreprotegidos les dificultan aprender otras estrategias más autónomas y recursos de independencia para lidiar con el ámbito social, particularmente el escolar.

Estas manifestaciones evaluadas cualitativamente son bastantes consistentes con las alteraciones psicoemocionales, evidenciadas psicométricamente. Además, uno de sus reforzadores, en especial, en las actitudes de dependencia, son las implicaciones de la epilepsia en la vida del paciente y su familia, en donde se observó sobreprotección. Krauskopf y De La Barra (2013) hacen mención de que el ambiente familiar y el estilo parental sobreprotector tienen un impacto considerable en los factores psicológicos y la calidad de vida.

La epilepsia es una enfermedad crónica, en la que se suele estigmatizar a quien la padece, Kirabira et al. (2018) expone una prevalencia elevada (34\%) de estigma en niños y adolescentes con epilepsia en su investigación y adiciona que el estigma es un problema importante a trabajar. Este hecho ha sido corroborado por las referencias de los pacientes y de sus padres, quienes han señalado cómo los otros niños rechazan al menor y cómo este manifiesta miedo e incluso percibe burlas. Es un padecimiento que impacta a toda la familia (Baki et al., 2004, Fayed et al., 2021; Jakobsen y Elklit et al., 2021) y que amerita un tratamiento multidisciplinario, para facilitar la adhesión terapéutica, incrementar su calidad de vida y prevenir el desarrollo de futuras patologías psicológicas/psiquiátricas. Para Krauskopf y De La Barra (2013), el tratamiento multidisciplinario de los pacientes con epilepsia es esencial, por lo que en Panamá se debe implementar un enfoque preventivo en salud mental, particularmente para los pacientes pediátricos con enfermedades crónicas.

Los resultados de esta investigación posibilitan el aumento en el interés ante el diseño de planes psicoterapéuticos de intervención para las alteraciones psicoemocionales evidenciadas, de modo que pueda prevenirse el establecimiento de patologías mentales que afecten al paciente. Así mismo, los aportes de la investigación resaltan la importancia de la psicología en el tratamiento multidisciplinario de la enfermedad y refuerza la necesidad de incluir los servicios de psicología en los diagnósticos de enfermedades crónicas pediátricas, como la epilepsia, en Panamá. Para futuros aportes investigativos, es necesario explorar en mayor detalle aspectos como la calidad de sueño en los niños y estilo de parentalidad de los cuidadores primarios, con el fin de generar planes psicoeducativos para padres, que prevengan el desarrollo de prácticas desadaptativas y perjudiciales para la salud mental de los niños panameños con padecimientos crónicos.

No obstante, cabe resaltar que se presentan algunas limitaciones importantes en este estudio como el diseño de investigación transversal, la reducida cantidad de participantes que dificultó la incorporación de análisis estadísticos de los resultados de mayor rigor, y, por lo tanto, los hallazgos no se pueden extrapolar con certeza a la población pediátrica en general con diagnóstico de epilepsia. Así mismo, no se consideran otros factores de confusión que podrían introducir sesgos en la etiología de la sintomatología como las condiciones socioeconómicas, experiencias durante la pandemia, diferencias en el uso de la medicación, foco de su epilepsia, pautas de crianza y presencia/ausencia de comorbilidades con otros trastornos del neurodesarrollo.

\section{Agradecimientos}

Al Hospital Materno Infantil José Domingo de Obaldía, mi gran reconocimiento, por su colaboración durante el transcurso de la investigación. A los profesores Mariluz Díaz, José Raúl Aparicio y Edgardo López por su asesoría en el desarrollo del trabajo. A la doctora Leidys Torres, por corregir el contenido, desde la perspectiva de idiomática. Igualmente, al doctor Jaime Estrella por sus valiosas orientaciones en el proceso de elaboración del manuscrito y publicación. 


\section{Referencias}

Asociación Americana de Psiquiatría (2014). Manual diagnóstico y estadístico de los trastornos mentales. Editorial Médica Panamericana.

Baki, O., Erdogan, A., Kantarci, O., Akisik, G., Kayaalp, L. y Yalcinkaya, C. (2004). Anxiety and depression in children with epilepsy and their mothers. Epilepsy \& Behavior, 5(6), 958-964. https://doi.org/10.1016/j.yebeh.2004.08.016

Beck A.; Rush, J.; Shaw, B. y Emery, G. (2010). Terapia cognitiva de la depresión. Editorial Desclée de Brower, S.A.

Bellak, L. y Sorel Bellak, S. (2007). Test de apercepción infantil con figuras animales (CAT-A). Editorial Paidós.

Bitsko, R.; Holbrook, J.; Ghandour, R.; Blumberg, S.; Visser, S.; Perou, R. y Walkup, J. (2018). Epidemiology and Impact of Health Care Provider-Diagnosed Anxiety and Depression Among US Children. Journal of Developmental and Behavioral Pediatrics, 39(5), 395-403. https://doi.org/10.1097/DBP.0000000000000571

Branden, N. (2007). Los seis pilares de la autoestima. Ediciones Paidós Ibérica, S.A.

Carballeda, J., Peláez, J., De Castro, M. y Montañés, F. (2018). Psiquiatría psicosomática del niño y adolescente con enfermedades crónicas: enfermedades renales. Revista de Psiquiatría Infanto-Juvenil, 35(4), 309-317. https://doi.org/10.31766/revpsij.v35n4a3

Cardoze, D. (2005). Escalas clínicas en psiquiatría juvenil. Editorial Universitaria Carlos Manuel Gasteazoro.

Centers for Disease Control and Prevention. (2020). Data and Statistics on Children's Mental Health. https://www.cdc.gov/childrensmentalhealth/data.html\#ref

Clark, D. y Beck, A. (2012). Terapia Cognitiva para Trastornos de Ansiedad: Ciencia y Práctica. Editorial Desclée de Brouwer, S.A.

Conde-Guzón, P.-A., Soria-Martín, C., Cancho-Candela, R., Quirós-Expósito, P., Conde-Bartolomé, P., y Bulteau, C. (2020). Parental report of quality of life in children with epilepsy: A Spanish/French comparison. Epilepsy \& Behavior: E\&B, 105. https://doi.org/10.1016/j.yebeh.2020.106968

Dal Canto, G., Pellacani, S., Valvo, G., Masi, G., Ferrari, A. R., y Sicca, F. (2018). Internalizing and externalizing symptoms in preschool and school-aged children with epilepsy: Focus on clinical and EEG features. Epilepsy \& Behavior: E\&B, 79, 68-74. https://doi.org/10.1016/j.yebeh.2017.10.004

Del Barrio, V., y Carrasco, M. Á. (2016). Problemas conductuales y emocionales en la infancia y la adolescencia.Padres y Maestros / Journal of Parents and Teachers, (365), 55-61. https://doi.org/10.14422/pym.i365.y2016.008

Devinsky, O. y Vazquez, B. (1993). Behavioral changes associated with epilepsy. Neurologic Clinics, 11(1), 127-149. https://www.ncbi.nlm.nih.gov/pubmed/8441366

Devinsky, O. y Vazquez, B. (2003). Epilepsy and anxiety. Epilepsy\&Behavior, 4(4), $20-25$. https://doi.org/10.1016/j.yebeh.2003.10.005

Fayed, N., Garrone, J. N., Russell, D. J., y Ronen, G. M. (2021). Quality of life (QOL) narratives of growing up with epilepsy from youth and family perspectives. Epilepsy \& Behavior: E\&B, 114(Pt A), 107613. https://doi.org/10.1016/j.yebeh.2020.107613

Fernández-Castillo, A., y Vílchez-Lara, M. J. (2015). Alteración emocional en atención primaria y urgencias pediátricas. ¿Se enfadan los padres durante la atención pediátrica? Revista latinoamericana de psicologia, 47(3), 205-212. https://doi.org/10.1016/j.rlp.2015.06.003

Ghandour, R.; Sherman, L.; Vladutiu, C.; Ali, M.; Lynch, S.; Bitsko, R. y Blumberg, S. (2019). Prevalence and Treatment of Depression, Anxiety, and Conduct Problems in US Children. The Journal of Pediatrics, 206(3), 256-267. https://doi.org/10.1016/j.jpeds.2018.09.021

Gómez-Restrepo, C., Ramirez, S., Tamayo Martínez, N., Rodriguez, M. N., Rodríguez, A., y Rengifo, H. (2016). Prevalencia de posibles trastornos mentales en niños con condiciones crónicas. Resultado de la Encuesta Nacional de Salud Mental Colombia 2015. Revista colombiana de psiquiatria, 45(1), $135-140$. https://doi.org/10.1016/j.rcp.2016.08.005

Howes, E.; Zwicker, A.; Uher, R. y Pavlova, B. (2020). Cognitive-behavioural interventions for prevention 
and treatment of anxiety in young children: A systematic review and meta-analysis. Clinical Psychology Review, 81. https://doi.org/10.1016/j.cpr.2020.101904

Isomaa, R.; Väänänen, J.; Fröjd, S.; Kaltiala-Heino, R. y Marttunen, M. (2013). How Low Is Low? Low SelfEsteem as an Indicator of Internalizing Psychopathology in Adolescence. Health Education \& Behavior, 40(4), 392-399. https://doi.org/10.1177/1090198112445481

Jakobsen, A. V., y Elklit, A. (2021). Self-control and coping responses are mediating factors between child behavior difficulties and parental stress and family impact in caregivers of children with severe epilepsy. Epilepsy \& Behavior: E\&B, 122. https://doi.org/10.1016/j.yebeh.2021.108224

Jones, J. E. (2014). Treating anxiety disorders in children and adolescents with epilepsy: what do we know? Epilepsy \& Behavior: E\&B, 39, 137-142. https://doi.org/10.1016/j.yebeh.2014.06.021

Karanja, S. W., Kiburi, S. K., Kang'ethe, R., y Othieno, C. J. (2021). Emotional and behavioral problems in children with epilepsy attending the pediatric neurology clinic at a referral hospital in Kenya. Epilepsy \& Behavior: $E \& B, 114(\mathrm{Pt} \mathrm{A})$. https://doi.org/10.1016/j.yebeh.2020.107477

Kirabira, J., Nakawuki, M., Fallen, R., y Zari Rukundo, G. (2018). Perceived stigma and associated factors among children and adolescents with epilepsy in south western Uganda: A cross sectional study. Seizure: The Journal of the British Epilepsy Association, 57, 50-55. https://doi.org/10.1016/j.seizure.2018.03.008

Kotwas, I., McGonigal, A., Bastien-Toniazzo, M., Bartolomei, F. y Micoulaud-Franchi, J. (2017). Stress regulation in drug-resistan epilepsy. Epilepsy \& Behavior, 71, 39-50. https://doi.org/10.1016/j.yebeh.2017.01.025

Krauskopf, V. y De La Barra, F. (2013). Trastornos psiquiátricos en los pacientes con epilepsia. Revista Médica Clínica Las Condes, 24(6), 979-985. https://doi.org/10.1016/S0716-8640(13)70252-9

Lacomba, L., Valero, S., Casaña, S., Pérez, M. y Montoya, M. (2018). Enfermedad crónica pediátrica: estudio comparativo de los niveles de ansiedad, depresión y autoestima. Revista de Psicología Clínica con Niños y Adolescentes, 5(3), 48-53. 10.21134/rpcna.2018.05.3.7

Lang, M. y Tisher, M. (2003). Cuestionario de Depresión para Niños. TEA Ediciones, S.A.

Larson, A.; Ryther, R.; Jennesson, M.; Geffrey, A.; Bruno, P.; Anagnos, C.; Shoeb, A.; Thibert, R. y Thiele, E. (2012). Impact of pediatric epilepsy on sleep patterns and behaviors in children and parents. Epilepsia, 53(7), 1162-1169. https://doi.org/10.1111/j.1528-1167.2012.03515.x

Liga Internacional contra la Epilepsia. (2017). Documento de posición de la Comisión de Clasificación y Terminología de la ILAE. https://www.ilae.org/files/ilaeGuideline/ClassificationEpilepsiesScheffer2017-Spanish.pdf

Lino, F.; Otero, P. y Blanco, V. (2019). Guía de intervención de la depresión. Editorial Síntesis, S.A.

López, V., Barragán, E., Hernández, M., Watemberg, N. y Legido, S. Calidad de vida en pacientes adolescentes con epilepsia en México. Rev Med Hondur, 78(4) (2010), 179-182. http://www.bvs.hn/RMH/pdf/2010/pdf/Vol78-4-2010-4.pdf

Márcia Targas, E. (2014). Evolución de la terapéutica medicamentosa de las epilepsias. En E. Márcia Targas; G. Contreras y L. Ríos (Eds.). Sao Paulo: Leitura Médica Ltda. https://www.ilae.org/files/dmfile/TratamientoFarmacologico-ALADE.pdf

Michaelis, R.; Tang, V.; Goldstein, L.; Reuber, M.; LaFrance Jr, W.; Lundgren, T.; Modi, A. y Wagner, J. (2018). Psychological treatments for adults and children with epilepsy: Evidence-based recommendations by the International League Against Epilepsy Psychology Task Force. Epilepsia, 59(7), 1282-1302. https://doi.org/10.1111/epi.14444

Ministerio de Salud. (2018). Boletín estadístico: anuario 2017. http://www.minsa.gob.pa/sites/default/files/publicacion-general/boletin_2017.pdf

Ministerio de Salud. (2018). Indicadores básicos de país. http://www.minsa.gob.pa/sites/default/files/publicaciongeneral/indicadores_basicos_de_salud_2017_.pdf

Morris, C. y Maisto, A. (2011). Introducción a la Psicología. Pearson Educación.

Moya, J. y Fernández, N. (2017). Depresión y suicidio en la infancia y adolescencia. Pediatría Integral, 21(2), 116.e1-116.e6.

http://www.codajic.org/sites/www.codajic.org/files/Depresi\%C3\%B3n\%20y\%20suicidio\%20\%20en\%2 0la\%20infancia\%20y\%20adolescencia\%20.pdf

Murk, C. (2013). Self-Esteem and Positive Psychology. Springer Publishing Company. 
Nadkarni, J., Jain, A., y Dwivedi, R. (2011). Quality of life in children with epilepsy. Annals of Indian Academy of Neurology, 14(4), 279-282. https://doi.org/10.4103/0972-2327.91948

Organización Mundial de la Salud. (2019). Epilepsia. https://www.who.int/es/news-room/factsheets/detail/epilepsy

Orth, U. y Robins, R. (2014). The Development of Self-Esteem. Current Directions in Psychological Science, 23(5), 381-387. https://doi.org/10.1177/0963721414547414

Ortuño-Sierra, J., Fonseca-Pedrero, E., Paíno, M., y Aritio-Solana, R. (2014). Prevalencia de síntomas emocionales y comportamentales en adolescentes españoles. Revista de psiquiatría y salud mental, 7(3), 121-130. https://doi.org/10.1016/j.rpsm.2013.12.003

Quesada, A., Justicia, D., Romero, M., y García, T. (2014). La enfermedad crónica infantil: repercusiones emocionales en el paciente y en la familia. Revista INFAD de Psicología, 4(1), 569-576. https://doi.org/10.17060/ijodaep.2014.n1.v4.832

Ramos-Loyo, J. y Sanz-Martin, A. (2005). Trastornos emocionales en la epilepsia. Revista Ciencia 56(3), 3139. https://www.revistaciencia.amc.edu.mx/images/revista/56_3/trastornos_emocionales.pdf

Roa, A. (2013). La educación emocional, el autoconcepto, la autoestima y su importancia en la infancia. Edetania: estudios y propuestas socio-educativas, (44), 241-257. https://revistas.ucv.es/index.php/Edetania/article/view/210

Rosenberg, M.; Schooler, C.; Schoenbach, C. y Rosenberg, F. (1995). Global self-esteem and specific selfesteem: different concepts, different outcomes. American Sociological Review, 60(1), 141-156. http://www.jstor.org/stable/2096350

Salari, R., Wells, M., \& Sarkadi, A. (2014). Child behaviour problems, parenting behaviours and parental adjustment in mothers and fathers in Sweden. Scandinavian Journal of Public Health, 42(7), 547-553. https://doi.org/10.1177/1403494814541595

Sánchez, P. y Cohen, D. (2020). Ansiedad y depresión en niños y adolescentes. Adolescere, 8(1), 16-27. https://www.adolescenciasema.org/ficheros/REVISTA\%20ADOLESCERE/vol8num12020/2\%20Tema\%20de\%20revision\%20$\% 20$ Ansiedad\%20y\%20depresion\%20en\%20ninos\%20y\%20adolescentes.pdf

Sattler, J. (2010). Evaluación Infantil: Fundamentos Cognitivos. Editorial El Manual Moderno.

Schraegle, W. y Titus, J. (2017). The relationship of seizure focus with depression, anxiety, and health-related quality of life in children and adolescents with epilepsy. Epilepsy \& Behavior, 68, 115-122. https://doi.org/10.1016/j.yebeh.2016.12.009

Seyfhashemi, M. y Bahadoran, P. (2013). Depression in Children and Adolescents with Epilepsy: a 15 Year Research Review of Prevalence, and Demographic and Seizure Related Correlates. Iranian Journal of Pediatrics, 23(1), 1-7. https://www.ncbi.nlm.nih.gov/pmc/articles/PMC3574984/

Spielberger, C. (1966). Theory and research on anxiety. En C. Spielberger. (Ed.), Anxiety and behavior (pp. 3-19). Academic Press. https://doi.org/10.1016/B978-1-4832-3131-0.50006-8

Spielberger, C., Edwards, C., Lushene, R., Montuori, J. y Platzek, D. (2009). Cuestionario de Autoevaluación Ansiedad Estado/Rasgo en niños. TEA Ediciones, S.A.

Varni, J., Katz, E., Seid, M., Quiggins, D. y Friedman-Bender, F. (1998). The pediatric cancer quality of life inventory-32 (PCQL-32) I. Reliability and validity. American Cancer Society, 82(6), 1184-1196. Doi: 10.1002/(sici)1097-0142(19980315)82:6<1184::aid-cncr25>3.0.co;2-1

Velarde, S.; Sánchez, E.; Manzané, F. y Britton, G. (2014). Prevalencia estimada de trastornos conductuales y emocionales en la población pediátrica en Panamá. Investigación y Pensamiento Crítico, 2(3), 35-52. $\mathrm{https}: / / \mathrm{www} \cdot$ google.com/url? $\mathrm{sa}=\mathrm{t} \& \mathrm{rct}=\mathrm{j} \& \mathrm{q}=\& \mathrm{esrc}=\mathrm{s} \&$ source $=\mathrm{web} \& \mathrm{~cd}=\& \mathrm{cad}=\mathrm{rja} \& u a c t=8 \& \mathrm{ved}=2 \mathrm{ahU}$ KEwjt3vGF3ojsAhXvpVkKHTJQC1YQFjABegQIBRAB\&url=http\%3A\%2F\%2Fipc.org.pa\%2Fojs\%2 Findex.php\%2Fipc\%2Fissue\%2Fdownload\%2F5\%2F9\&usg=AOvVaw36Y-dD_ZxSF5AGX3ggFu0p

Wei, Z., Ren, L., Wang, X., Liu, C., Cao, M., Hu, M., Jiang, Z., Hui, B., Xia, F., Yang, Q., Liu, Y., y Deng, Y. (2021). Network of depression and anxiety symptoms in patients with epilepsy. Epilepsy Research, 175. https://doi.org/10.1016/j.eplepsyres.2021.106696

Zambrelli, E., Turner, K., Vignoli, A., La Briola, F., Dionisio, S., Malanchini, S., Galli, F., y Canevini, M. P. (2020). Sleep disturbances in Italian children and adolescents with epilepsy: A questionnaire study. Epilepsy \& Behavior: E\&B, 106. https://doi.org/10.1016/j.yebeh.2020.107014

Zurita-Cruz, Wakida-Kusunoki, Domínguez-Camacho, Padilla-Rojas, Maldonado-Rivera y Peña-Pelayo. 
(2017). Alteraciones psicoemocionales en adolescentes con obesidad. Revista Mexicana de Pediatría, 84(5), 176-181. https://www.medigraphic.com/pdfs/pediat/sp-2017/sp175b.pdf. 